washing out the bowel with plain saline or mild astringent solutions, such as $\frac{1}{2}$ per cent. argyrol or protargol, is often more successfal than anything else.

(3) Ulcerative Colitis.

Ulceration of the colon has become well recognized in recent years as a not uncommon cause of obstinate and chronic diarrhoea. In many cases the disease has originated in an attack of tropical dysentery, which the patient has never properly got over, but a certain number are met with in patients who have never been ab. yad. The relation of such cases to true dysentery is a matter which I do not propose to go into, as it is still the subject of dispute, and in any case is more of theoretical than practical importance. The stools in cases of ulcerative colitis, whether dysenteric or non-dysenteric in origin, tend to have more of the true colonic character than in any other form of diarrhoea, blood and mucus being usually present, whilst tenesmus is not infrequently complained of. One is aided in the diagnosis by a history of dysentery, but certainty can only be reached by the use of the sigmoidoscope.

The medical treatment of these cases is apt to be unsatisfactory, but in the milder cases one may achieve success by the use of the measures employed in catarrhal cases (see above). I have sometimes also had brilliant results from the prolonged use of an exclusive diet of peptonized milk. Vaccine treatment also has a limited sphere of usefulness, but chiefly as an aid to other methods; it is most likely to be successful when cultures yield an almost pure growth of some organism foreigi to the bowel-for example, the pneumococcus. In cases in which there is a fairly recent history of acute dysentery ipecacuanha is worth a trial. It was brilliantly successful in the following case:

$\Lambda$ lfred N., aged 35, a soldier, returned from India in December, 1906, and in February, 1907, began to suffer from diarrhoea, with the passage of blood and mucus, and attended by tenesmus. He had had several attacks of dysentery, not only in India, but also in Egypt and South Africa. No amoebae or dysentery also in Egypt and South Africa. No amoebae or dysentery He was given pulverized ipecacuanha in large doses, beginning with 30 grains, the usual precautions being taken against vomiting, with the result that the diarrhoea immediately ceased and the motions became normal. He left the hospital apparently quite well.

In most cases of ulcerative colitis, however, whether dysenteric in origin or not, I believe appendicostomy to be the best treatment. How successful it may be the following cases show:

Mr. M., aged 34, came under observation in April, 1910. He had an attack of dysentery in Hong Kong in 19j5, and in September, 1909, underwent an operation for liver abscess in Borneo. Diarrhoea ensued after this and had continued since. He had been treated with ipecacuanha in America without success.

The stools numbered about five daily, some of them containing blood and mucus. Abdominal examination elicited slight thickening and tenderness of the sigmoid. The sigmoidoscope
showed the mucous membrane as high as it could be reached to be congested, covered with glairy mucus, and studded with numerous smaH superficial ulcers. Appendicostomy was performed by Mr. Furnivall, and the colon washed out daily with normal saline. Seven weeks later a second examination with the sigmoidoscope. showed that the mucous membrane was entirely healed; the patient had lost his diarrhoea and had gained $2 \frac{1}{4}$ st. in weight.

In the following case the benefit of the operation was even more striking:

Colonel E., aged 68, was first seen in July, 1907. In 1894 he left India on account of dysentery. On his return home he was treated with ipecacuanha and remained well for two years. The diarrhoea then returned and had persisted more or less ever since, in spite of treatment by various astringent medicines, by all sorts of diets, and by a course of Plombières douches.

On examination he was found to be anaemic but fairly nourished. The motions were small, frequent, extremely offensive, and contained blood and mucus; they were passed with much straining. The sigmoidoscope showed the colon to be studded with small ulcers for a distance of $20 \mathrm{~cm}$. above the anus. Appendicostomy was performed and the colon washed out, first with saline and afterwards with weak protargol solution. Eighteen months later he wrote that he wae perfectly well except for a slight tendency to constipation.

\section{Nervous DiarRHoEA.}

By nervous diarrhoea one means that form of chronic looseness of the bowels in which no organic disease or secretory defect is to be detected in the alimentary canal but in which the disorder appears to result from an exag. gerated irritability of the nerve mechanism which controls peristalsis. Two types can be recognized. In one the action of the bowels tends to take place immediately after or even during meals. To this type the term "postprandial". or "lienteric" diarrhoea is usually applied. It is specially common in children. The fault here appears to consist in an exaggeration of the normal reflex stimulation of peristalsis which always takes place to a slight degree when food enters the stomach. In the other type the diarrhoea is more affected by emotional impressions. Thus, in one of my patients, a harassed, over-driven business man of anxious temperament, it always tended to come on if he was so situated that he could not easily retire for an action of the bowels-for example, if he was travelling in a railway carriage without a lavatory compartment. In another, a society lady, the trouble was always most assertive whenever she had to attend some specially important function. I have also known it affect a nervous anaesthetist (a lady) whenever she had to give an anaesthetic for a particular and rather exacting surgeon, the sound of the latter's step on the stair leading to the operating theatre being enough immediately to induce an attack.

It is possible, I think, that in some of these cases there is really an underlying degree of catarrh of the colon, which causes the latter to respond forcibly to nervous impulses, which in a healthy individual would only be sufficient to produce a mild stimulation of peristalsis.

In the treatment of the nervous form of diarrhoea not much can be effected by diet, and in any case the condition is one of such chronicity that the patient has usually found from his own experience what it is well to avoid. In the lienteric tyl. small doses of opium before meals act almost as a specific. I know that arsenic and nux vomica are often recommended in such cases, but for my own part I have found opium far superior to them in its power of controlling the disorder. In the emotional type opium should be reserved for use in emergencies and to enable the patient to have confidence in his bowels for particular occasions, whilst one should endeavour to allay the general condition of nervous irritability which underlies the condition by regulating the whole life and by the use of general sedatives, such as the bromides.

REFERENCES.

1 Scottish Medical and Surgical Journal, xiv, 1904, p. 321. 2 See 8 1 Scottish Medical and Surgical Journal, xiv, 1904, p. $321 .{ }^{2} \mathrm{~S}$
paper by the author on Coeliac Disease, Practitioner, August, 1911.

\section{THE RELATIONS OF BIOLOGY TO PATHOLOGY.}

By HASTINGS GILFORD, F.R.C.S., READING.

"We have viewed every anatomical and pathologic part as an entity, and man as an isolated phenomenon in Nature. May we not find in the laws of phvlogeny and association the master key that will open to us the explanation of many of the pathologic phenomena as they havealready explained many normal phenomena? And may medicine not correlate the pathologic phenomena of the sick man with the forces of evolution, as the naturalists have correlated the phenomena of the sound man, and disease as well as health be given its evolutionary setting?"-Crile, BRITISH MEDICAL JouknaL, 1911, vol. ii, p. 945.

IT seems at first quite preposterous for any one to assert that the work of Pasteur and of Lister has in some respects had a retarding effect upon medical science. But that such is the case can, I think, be demonstrated beyond question. So strong has been the light which has been shed by the two great luminaries upon surgical pathology and upon some departments of medicine that other and quite as important departments have been thrown into the shade. We all know how Pasteur and Lister in more than one sense revived medicine and surgery, much as Schwann revived our conceptions of anatomy, and Virchow of morbid anatomy. Before the discoveries of Pasteur and of Lister surgery had arrived at a stage from which it could not advance. It had become stagnant or chrysalised. From this hidebound condition it was liberated as by an act of obstetrics into a far freer and more abundent life than it had ever known before, and the instrument by means of which this was accomplished was biology. And 
not only was surgery biologized, but our ideas of pneumonia, of rheumatic fever, of tuberculosis, of syphilis, and of many other diseases have undergone similar emancipation.

Nevertheless, in spite of, as well as because of, this great and beneficent change, a large group of diseases still remains in almost as benighted a state in respect to our knowledge of its pathology as it was in the Sixties. That our understanding of bacteriology, instead of enlightening our darkness in regard to these diseases, has tended to their obscurity is shown in many ways, perhaps in no respect more emphatically than by the present status of clinical medicine as a science in comparison with bacteriology. The nomenclature which has cropped up about the new science is also eloquent of the same loss of prestige. Let us take for example the word "research." What can be more arrogant than the modern use of this word. It is applied almost exclusively to investigations into the minuter or more subtle forms of matter. Hence, he who undertakes a test tube investigation of the plague is a " researcher," and is employed in a " research," but he who does equally diligent work on the clinical aspects of, say, Bright's disease, or Hodgkin's disease, has no such dignity bestowed upon him. This use of the word "research" is based upon the tacit assumption that in the one case the worker is engaged in a veritable pioneer exploration, in the othar he is not. However good the result of the clinical study may be to the individual, so far as the advancement of learning is concerned it is about as profitable as walking on a treadmill or flogging a dead horse.

There are other words or phrases which point even more definitely to the overwhelming predominance of bacteriological over clinical science. These are words or phrases appropriate to microbial disease, but sometimes forced out of their proper place to do duty in the non. micröbial disorders. "Toxins of bacterial origin," " toxaemia of pregnancy," "autointoxication," " toxaemia," or "toxin" itself is full of meaning when appropriately applied in microbial disease, but is vapid, meaningless, and worse than useless as commonly applied to diseases other than microbial. Thus, when acute rheumatism or gonorrhoeal rheumatism is described as an intoxication a more apt and fitting word can hardly be imagined, for it is exactly appropriate to the circumstances. But when arthritis deformans is described as an intoxication, or, still worse, autointoxication, the term as we commonly use it is significant of nothing but a sort of mental flatulency on the part of the user or as a substitute for thought. In the one case the word rings true, as of a genuine coinage; in the other it is as flimsy, as unsubstantial, as a paper currency. Moreover, it has no basis of fact, and represents nothing save a bankrupt exchequer. Being at a loss to account for a disease, and having found bacteriology of so great value when properly applied, we think we cannot do better than foist off a bacteriological term in the hope that it may be accepted as a genuine explanation.

Much the same may be said of the use of these same words to such conditions as cirrhosis of the liver, pernicious anaemia, leukaemia, Graves's disease, progressive muscular atrophy, osteomalacia, muscular dystrophy, cancer. A toxin may in some cases give the malady its first impetus, or possibly may appear as an epiphenomenon in its course, but nothing seems clearer than that the morbid process is not toxic in nature, seeing that it may arise, run its course, and terminate under circumstances in which the toxins seem to play no part whatsoever.

The question now arises: If the disorders alluded to are not the work of micro-organisms, what is their pathology? It is hardly within the scope of this article adequately to answer this question, even if it be possible. One can go no farther than to ask that as surgery, midwifery, gynaecology have been so gloriously revived as the result of biology, ought we not to make use of a similar key in order to unlock the mysteries which are still concealed behind the names of diseases of unknown pathology, some of which have just been mentioned? Moreover, each and every disease of this group has been so often and so persistently regarded as a bacterial product, and with so little success, that it is surely time that we abandoned this department. of biology and directed our attention to some other way of accounting for them. That' they can be accounted for in another way is, I think, capable of proof. Fortunately, this proof requires no output of genius. All that is required is the use of an average intelligence, a little patience and ingenuity, the resolve to think biologically, not pathologically, and, above all, the ability to purge the mind of those preconceptions and prejudices which do more than anything else to obscure new issues. So equipped, and with the way clear, we find all the materials we require ready at hand and only waiting to be put together in their proper order.

These materials are the common biological facts of variation, heredity, adaptation to circumstances, correlation, interwoven in their proper order with the fleeting changes of age, or with the pcculiarities incidental to sex. The doctrines of reversion and of the recapitulation of characters, the facts of comparative anatomy, of anthropology, of palaeontology, must be liberally drawn upon, and adequate allowance must be made for the different effects of similar circumstances upon single cells, upon cell communities or organs, and upon the man as a whole.

In the course of this inquiry we shall speedily realize to the full the reality of the gulf which separates the two groups. In the one group the component disorders are marshalled into systems and organized into a science; in the other group we see little more than a rabble of almost disconnected diseases. These latter do not as yet display any of that orderly arrangement of knowledge which constitutes a science ; for, though it is true that their structure has been, and is being, worked out scientifically, this cannot be said of the diseases themselves. Their anatomy is scientific, but not their pathology.

We next find that the diseases in question differ radically from the microbial diseases, in that while the microbial disorders are the products of external agents, each member of this scattered loorde is of intrinsic production, or, more accurately, the members of the one group are of contemporary and of the other group of prehistoric origin.

Our next step is to realize that this second group may be divided into two further gronps. In the on $\epsilon$ the disorders are solely of intrinsic (or remoter) production; in the other they are fundamentally intrinsic, but are also in part the outcome of environment. To realize the significance of this division we must go to the doctrine of variation.

Biological variations have been divided by Bateson into two kinds-namely, those which consist in numerical changes, as in polydactylism, and those which consist in an alteration in constitution. It is the latter with which we are now concerned. Of these substantive variations some are more emplatic, spring into being with a leap, irrespective of environment, and are variously termed mutations, sports, discontinuous variations. These are handed down from generation to generation, unless of such a nature as to provoke sterility, or in other ways to facilitate their own extinction. Of this form of variation albinism is a good example.

The second kind of variation is less pronounced, is never hereditary, and is amenable to the influence of environment. Hence it is dependent for its existence partly upon intrinsic and partly upon extrinsic causes. This minor order of variation is termed fluctuation or continuous variation. It is this variation which accounts for the whiteness of the Arctic fox. Evidently, therefore, certain wild animals are liable to become affected with whiteness. The one form of whiteness is erratic in its appearance, or, in other words, occurs sporadically, without reason, and animals so affected are prone to transmit the abnormality to their descendants. This is a mutation. The other whiteness comes about partly as the result of an occult factor, termed fluctuation, and partly as the result of an environment calculated to harmonize with this particular form of variation, but is never the result of heredity.

We are so accustomed to regard genetics and $\epsilon$ volution in general from the utilitarian point of view as making for the progress of the race, that we ignore and even forget that as "everye white will have its blacke and everye sweete its sowre," so also the very doctrine of the survival of the fit implies that there are also the unfit. It is in this evolutionary scrap-heap that we must make our search for the variations which are diseases. These diseases consist essentially in extremes. For example, a certain brain area is too small or too big, too tardy or too hurried in its development. Either of these extremes is abnormal, sufficiently abnormal, it may be, to constitute disease, and to cause the individual to whom the brain belongs to be 
cast on one side as one of the unfit. So after the same fashion with other organs, with individual cells, or with the whole human being. Each division is liable to these variations. Moreover, we have no difficulty in separating from our group of diseases some which are of pronouncec degrce, spring into being and run their course to all appearances spontaneously, are sporadic, and apt to occur hereditarily. Among these are sporadic cretinism, achondroplasia, idiopathic pernicious anaemia, idiopathic microcephaly, muscular dystrophy, hypertrophic cirrhosis of the liver, osteitis deformans, Friedreich's ataxia. These correspond with the major or discontinuous variations. They are biological mutations rendered pathological by being carried to extremes.

Other disorders show no tendency to heredity, occur partly as the result of some occult influence, and partly as the result of definite causes. These correspond with the fluctuations or minor variations. Among them are endemic cretinism, rickets, atrophic cirrhosis of the liver, chlorosis, and those forms of pernicious anaemia-microcephaly, osteomalacia, and osteitis deformans - which can be traced to the operation of causes. These are in trutl of a double origin, for though they originate as variations, their actual appearance is due to the influence of a pernicious environment.

But it is evident that we shall have to enlarge our views of that word "environment." We cannot restrict the word to those circumstances which actually surround the human being, but must also extend it to those things which he takes internally, for it must be obvious that, though not strictly speaking the envirohment of the whole man, by circulating in his fluids they constitute an environ. ment to his organs and cells. Hence, the poisonous gin which a man swallows at the bar of a low public house is just as truly a part of his environment as are the foul air, the coarse and dirty furniture, and the evil talk of his ragged and grimy associates. So also in respect to endemic cretinism, the environment which starts it into being may be in part some gloomy valley in which such cretins so often live. It may also in part consist of the sedimentladen glacier water which constitutes their daily drink.

We all understand that the normal evolution of a healthy human being is facilitated by a congenial environment. An abundance of good food and of fresh air, comfortable clothes, a temperate climate, cheerful society, good music, paintings, and literature, all tend to soundness of health and to vigour of mind and body. So, also, inadequate fooc and clothing, over-fatigue, the stale air of unventilated rooms, paucity of sunshine, and poverty, misery, hardship, or any form of mental shock or depression, however produced, all tend to lower the vitality and to facilitate, and in some degree to initiate, disease. The circumstances in the former case act congenially upon normal variations of minor degree to promote erolution. Circumstances in the latter case act congenially upon those variations which are decadent, reversionary, or regressice, tending to throw the man or some part of the man seriously back in the evolutionary scale, causing cells or organs to be at discord with the rest of the body or the man with other men. The one set of circumstances induces biological variations, the other pathological. Under the heading of a pernicious environment, we must include all those substances which taken into the body act inimically upon its con stituents, and have, therefore, received the special name of toxins. Though different in kind, these are fundamentally of the same nature in regard to their effects as the circum stances which have just been mentioned. All are indeed toxins, no matter whether they surround the body, are taken internally, are physical or psychical. Failure in business, disappointment in love, conspicuous ingratitude, the depravity of a near relative, the shock of a railway accident, or a serious local injury acts just as truly as a poision upon the body as does scarlet fever, enteric fever, or even alcohol or syphilis. The symptoms prcduced by an unexpected financial reverse may consist in sleepless ness, loss of appetite, depression of spirits, and a haggard expression of face; and those produced by scarlet fever may consist in fever, loss of appetite, sore throat, and a skin rash, but the differences are differences in detail, and do not affect the main proposition that both are poisonous and capable of acting in conjunction with latent variation to set up a state of intrinsic disease. And this secondary intrinsic disease, say Graves's disease, myxoedema, or rheumatoid arthritis, once established, will continue even if the extrinsic influence be completely withdrawn.

We have now arrived at a point from which we can reconcile our conception of normal evolution with our conception of certain kinds of disease. In both cases we have to do with variations, major or minor. In the one the variations synchronize with circumstances which tend gradually to raise the individual in the scale of creation. In the other the variations are incompatible with normal evolution. They represent biological extremes and are, therefore, discordant. Under ordinary circumstances they remain latent or are suppressed; but if perchance the en. vironment should be of such a nature as to co-operate with or to accentuate them they cease to be latent, but become patent and show themselves in the form of disease.

Pathological variations may be either progressive or regressive. In other words, those variations which ordinarily mark the progress of a thing or of a quality may be exaggerated to such a degree as to cease to be normal, or may even come within the definition of disease. A good example of this kind of variation is the overgrown thyroid of Graves's disease The other variation is pathological and regressive, because it represents a thing or quality which has so fallen short of its normal standard as to constitute disorder. Thus microcephaly is a regressive variation, seeing that the growth of the brain is arrested long before it has reached a point which is compatible with healthy standards of 'brain development.'

From this it will be gathered that pathological variations are no more than the extreme forms of biological variations. They are pathological not because they are extremes, but because they are discordant. Thus the ears of a lop-eared rabbit are almost certainly the result of a variation of extreme degree, but no one can say they are pathological; for they happen to suit the taste of rabbit fanciers, and though they must be a nuisance to the rabbit, and must interfere with hearing, they tend to prolongation of life and the continuance of the race, and cannot therefore be regarded as morbid or out of place. So, also, when the "caudal appendage" of a dog is, so to say, curtailed as the result of a major variation, as in the bobtailed sheep dog, the result can hardly be termed either a deformity or a disease. And even when the limbs of a dog are shortened by that particular form of variation which we term "achondroplasia," the result is in no sense a distemper. If the dog were a wild dog, it would no doubt have that effect, seeing that it would make it more difficult for the animal to seize its prey, and wonld render it more liable to be killed by its enemies. But under circumstances now natural to this particular breed of dogs the achondroplasia, so far from unfitting the animal for existence, constitutes a positive advantage, and is a biological asset. The old turnspit dog, the dachshund, the Dandie Dinmont, the Sussex spaniel are all achondroplasic. Though a variation of major degree the achondroplasia is not pathological, seeing that it promotes longevity and tends to the perpetuation of the species. But when a human being is affected with achondroplasia we have to do with a major variation which is pathological, for, other things being equal, the shortness of the limbs, the deformity of the pelvic bones, and the other concomitants of achondroplasia constitute disabilities which both seriously handicap the individual in his progress through life and tend to bring about his racial extinction. Not only are many occupations barred against him because of his incongruous appearance, but he finds it difficult to secure a mate. Moreover, if the achondroplasic chance to be a woman, owing to the deformity of her pelvis she runs peculiar risks at the time of childbirth. Heuce for these reasons achondroplasia, which constitutes a normal condition in the dog, is very rightly regarded as a disorder in the man. It harmonizes with the circumstances natural to the dog, and is discordant with the circumstances natural to the man.

Another variation of major degree and progressive in character which is very decidedly pathological is that of the thyroid gland in Graves's disease. In this disorder the excessive growth of the thyroid is so obviously out of keeping with bodily circumstances in general that no one hesitates to call it a disorder. And so also with ill-development or premature degeneration of the thyroid gland, a major regressive variation which gives rise to sporadic cretinism. 
These are examples of major variation. An example of minor variation akin to achondroplasia is furnished by rickets, Rickets is due in the first place to some hidden factor. It is this which accounts for the fact that under similar circumstances the disease occurs in some children and not in others. This hidden factor is, of course, the variation or fluctuation. This variation is brought to the surface or fostered by certain circumstances which are favourable to the variation but are adverse to the individual. These circumstances are stale air, thin condensed milk scandalously deficient in fats, and all those other circumstances which we know so well as the causes of rickets.

In some disorders the distinction between major and ninor variation is not so marked. This is the case with rheumatoid arthritis. Rheumatoid arthritis or arthritis deformans is a disorder of whose pathology we are at present in complete ignorance and can do no more than conjecture. In the words of Dr. McCrae in the System of Medicine of which he is the joint editor,

There is " no agreement as to what the disease essentially is. To recount the various theories in detail is not necessary. relationship of rheumatism and gout, an inherited arthritic diathesis, a special kind of degeneration, disturbed nutrition, an inflammatory process, of essential nervous origin, due to injury, reflex irritation, or disease of a supposed joint centre, and some form of infection, have all been suggested at some time or other."

Now let us regard it as a variation and we see how much more precise, how much more just and satisfactory, is our conception of its pathology.

Arthritis deformans is a degeneration of the joints which may be divided into two forms. In the one it arises without ascertainable cause, chiefly in young people, is remarkable for the severity of the damage it inflicts, and for occasionally showing the influence of heredity. As no cause can be found so also is there no remedy, for though it runs a variable course it terminates almost inevitably in the complete ruin of the joint. This form is a mutation.

The other form may or may not arise spontaneously, yet in all cases we can detect the influence of a cryptic element accounting for the way in which, other circumstances being equal, the disease'seems capriciously to select some individuals in preference to others. It is a fluctuation, and like all fluctuations, responds to the influence of environment; but the fluctuation being of a decadent nature and having a tendency to thwart or reverse the normal course of evolntion this favourable environment is one which is calculated to lower vitality or accelerate old age. Overwork of the joint, anxiety, mental strain, direct damage by dislocation or other injury, the debilitating effect of previous illness, such as influenza, enteric fever, are all favourable to the appearance and continuance of the disease. Probably in most cases the general debility produced by bacterial intoxication constitutes in itself an environment sufficient for the purpose, but in some cases it is possible that a toxin acting locally in concentrated form, as in gonorrhoea or in rheumatic fever, is still more effective. But of all the circumstances favourable to the appearance of rheumatoid arthritis none can be compared with old age.

Though the disease itself is neither an inflammation (arthritis) nor an intoxicstion it is highly probable that the resisting power of the joints to local invasions is lowered by the morbid process and that they are therefore more liable than are sound joints to become inflamed.

It is the disease par excellence for the miracle worker and for the charlatan, for, though induced or encouraged by depressing surroundings, it is capable of being improved or even removed by influences of a contrary nature. The environment of worry or misery, which is in part re sponsible for the distemper, or the depressing conviction of being a cripple for life, which tends to its continuance is counteracted by the joyful and confident anticipation of a speedy and permanent cure, such as no one but the religious enthusiast or unprincipled quack is able to infuse into the victim of arthritis deformans. At the same time, it must be understood that this is not written for the sake of upholding such methods. Though it would be idle to deny their efficacy, it is certain that they are neither proper nor expedient. They may answer for the time being, but a more rational and truthful way must be more successful in the end. A doctor cannot impress the imagination of his patients by festooning his rooms with crutches, by unscrupulous promises, or by adopting the blatant methods of the quack; yet, if he once realize the importance of a sanguine ontlook and of treatment by suggestion, he can enthuse the patient with his own optimism, and can offer that encouragement which is born of knowledge and experience. These must be far more effective in the long run than any form of exaltation or make-believe, however sensational.

It cannot be pretended that this is an adequate explana. tion of arthritis deformans, yet it is satisfactory so far as it goes, and may serve as an example of the way in which disease may be approached from its biological aspects. The next step is to inquire into the meaning of variations in general and of pathological variations in particular.

It is of course impossible to furnish such explanation within the limits of this article, but those who wish to inquire further into the nature of variations cannot do better than go to the Mneme theory of Semon. ${ }^{1}$ The value of this theory has not yet been fully realized by biologists in this country. Yet German authorities regard it as of momentous and far-reaching significance, and Haeckel has gone so far as to describe it as "the most important advance that evolution has made since Darwin, and the most valuable amplification of his theory of selection." 2 Semon translates the facts of heredity and of variation into terms of memory. As the brain cell is the seat of individual memory, so is the sex cell the seat of racial memory. Racial memory begins with the union of the sex cells. This act by a species of mnemonics furnishes the clue to the next step, just as in brain memory one event by association of ideas recalls another event. Racial memory once set going continues automatically until, on the attainment of middle age, the building of the body is completed. Racial, like ordinary, memory is not infallible. The registration of impressions received in the remote past, when animal evolution was beginning, are very imperfect, so that only the dim outlines of great and widespread changes are repeated. But as the construction of the body approaches completion impressions are reproduced with ever-increasing fidelity. Impressions may be blurred in one case and not in another, and occasionally actual lapses occur, so that important evolutionary events are forgotten. On the other hand, impressions may stand out far too conspicuously, to the derangement of the general effect. It is after this fasbion that variations, normal and pathological, arise. An act of racial forgetfulness, if of extreme degree, is a regressive major variation. The memory of a great racial disaster or depression may be so subdued by lapse of time as to remain latent, but the associations furnished by a depressing environment are capable of awakening those long dormant impulses; the memory of the ancient environment is revived by a modern environment of a similar tendency, and the two together give risc to a regressive minor variation.

These meagre outlines of part of a very comprehensive theory may serve to suggest how other kinds of variation may be explained. Perhaps the greatest achievement of the Mneme theory is that, through memory, the theory of evolution is brought into line with the undulatory theories of light, of heat, of electricity, and of other forms of energy.

The nature of the particular variations to which reference has been made can only be understood on inquiry into the principles which underlie growth, development, old age, and the other ingredients mentioned at the beginning as requisite for our biological composition. It is necessary to repeat that this explanation of pathological variations makes no pretence to completeness. All that one can hope to do within the pages of a weekly journal is to indicate, however imperfectly, the beginning of the way in which a host of diseases of unknown pathology may be reconciled with ordinary biological conceptions. Any one who will take the trouble to continue along the same path and will deal after a similar fashion with other diseases of the group will, I am convinced, reap an ample reward, and in the end will possess an understanding of their pathology comparable with that with which we now regard microbial disease. He will find that these disorders which are now among the mysteries of medicine will be no longer mysterious. He will under stand the "nature of cancer, of pernicious anaemia, of leukaemia, of Graves's disease, as we now understand 
that of syphilis, of malaria, and of tuberculosis. Disorders of this sort, like the microbial disorders, will naturally arrange themselves into groups; yet it will be found that all, in the words of Erasmus Darwin, can be brought within the fold of a "theory founded upon Nature, that should bind together the scattered facts of medical knowledge, and convert into one point of view the laws of organic life.";

\section{REFERENCES.}

1 Die Mneme als erhalten des Prinzip im Wechsel des organischen Geschehens. 2 Darwin and Modern Science, p. $142 .{ }^{3}$ Zoonomia, or the Law's of Organic Life, second edition, 1796, preface, 1). 2.

\section{ULCEROUS LESIONS OF THE TONGUE.} BY

J. HOWELL EVANS, M.A., M.B., M.CH.OxoN., F.R.C.S.ENG.,

SCRGEON TO THF PRINCE OF WALES'S GENERAL HOSPITAL, N. : LATE HCNTERIN PROFESSOR OF SCRGEIY, ROYAL COLLEG OF SCRGEONS OF ENGLAND, ETC.

[With Coloured Plate.]

WhEN every means at our command has been employed there should be no érror in the diagnosis of, and consequently no difference of opinion as to the nature of, any ulcer of the tongue. We may, from a clinical standpoint consider ulcers of the tongue as: $(a)$ Evidently simple, (b) definitely malignant, $(c)$ doubtful; but to delay the justifiable removal of a malignant ulcer or to excise a noncarcinomatous tongue are errors which will eventually carry with them their own condemnation, because they can be obviated if the precepts laid down in the following pages are closely adhered to and if the fallibility of clinical diagnosis is constantly borne in mind.

The "simple ulcer," recent in appearance and of obvious causation-for example, a sharp tooth, local trauma; or glossitis--is characterized by absence of neuralgic pain, absence of peripheral induration, and, though tumefaction may not be evident to the naked eye, a certain amount of local oedema is distinctly seen by means of a lens; further, there is an absence (?) of lymphatic enlargement, an absence of foul deposit on the neighbouring teeth, except that which may be accounted for by delay or neglect of cleantiness.

When the exciting causes are removed-projecting stumps or edges of teeth-or in the case of aphthous sores after due attention to gastric conditions, the general health, and salivary lithiasis, the simple ulcer invariably heals in a few days; if not, there is cause for grave suspicion.

Treatment.

1. Medicinal, by mouth washes, for gastritis or for infective folliculitis.

2. Dcntal, for removal of any projecting or irregular tooth; "no dentist should take any responsibility in the treatment of any ulcer of the tongue beyond the limited domain of his qualification as a dentist."

3. Topical, by chromic acid solution; by caustics (a) actual cautery, (b) thermo-cautery-Pacquelin, (c) electro-cautery, $(d)$ chemical caustics; by radium.

There is always a temptation to treat ulcers of the tongue by means of caustics, but this is a dangerous proccdure, owing to loss of time and inefficiency, and should rarely be followed. The treatment by the application of caustics, such as silver nitrate, can do no possible good if the lesion is cancer, though in my opinion its application to simple ulcers or other lesions cannot induce carcinoma.

Whatever radiotherapy may do, such application must be topical, like all other thermal and caustic remedies. Some may object to the application of radium to ulcers, lest its effect be no better than a caustic, and valuable time be lost in the course of an undiagnosticated carcinoma; the effect of radium is beneficial in cancer, and while causing healing superficially it likewise penctrates to a considerable depth.

The practitioner in all cases of soreness of the tongue or mouth must not forget that salivary lithiasis or ptyalo. lithiasis is of more frequent occurrence than is anticipated, or, in other words, that many conditions of the tongue are due $t$ s salivary constituents, and not to gastric conditions.
Treatment includes cleansing of teeth, compressed-air jet containing a spray, revolving brush for removal of tartar, facial massage of the salivary glands; drinking of waters; fruit, especially oranges and pineapple.

Tuberculous Ulcer.

The taberculous ulcer, situated generally on the dos'sum or at the tip of the tongue, is readily recognized as a pallid solitary sore, often of considerable depth with sharply cut edges, indolent base usually devoid of induration, and almost invariably associated with pulmonary or laryngeal tuberculosis in young adults. With such associated manifestations the lesion is recognized with no great difficulty, but when the ulcer occurs primarily on the tongue the question is more difficult from the clinical side.

To the naked eye the bottom of the ulcer may appear to remain stationary, except for extension of its satellitic foci, and cause but little if any inconvenience, yet resisting all treatment except caustics, cantery, or curctte ; curettage is the ideal treatment. Its slow progress is, however, rendered apparent if examination is closely made with the aid of a good lens.

If an ulcer is suspected of being tuberculous the following considerations should be kept in mind as having a definite bearing on the diagnosis, treatment, and prognosis -namely, age and existence of tuberculosis in other parts of the body.

I will refer to the more recent methods which may assist when doubt or aversion to the use of the knife exists.

Tuberculin Tests.-The more recent modifications of the tuberculin test evolved from the original injection method have led to its more extensive employment. and have given to us a fair idea of their respective values. The following are now extensively employed:

1. The ocular instillation test, or Calmette's ophthalmic reaction. A drop of tuberculin (T.O.A.) is instilled into the conjunctival sac. In twelve to twenty-four hours an inflammatory reaction results in tuberculous patients.

2. The skin inunction test, or Moro's percutaneons reaction. Equal parts of tuberculin (T.O.A.) and hydrated lanolin are rubbed into the skin, when a similar reaction to von Pirquet's will result.

3. The cutaneo-inoculation test, or von Pirquet's reaction The skin of the forearm, cleansed and scarified, is inoculated with a drop of tuberculin (T.O.A:). In about twenty-four hours a reaction occurs followed by a characteristic papule or vesicle.

The wider use of these methods during the past few years has impressed me with their clinical limitations. The results fall considerably short of the requirements for an ideal diagnostic method in suspected tuberculosis, though relatively good in confirmation of the clinically tuberculous.

The diagnosis demands the employment of:

1. Tuberculin tests.

2. Scrapings of the ulcer (epithelioid cells and tubercle bacilli).

3. Excision of a piece (epithelioid cells, tubercle bacilli, and giant cells).

Scrapings are unsatisfactory, as by this means oftentimes only the detritus of necrotic cells is obtained; as in the case of the tongue a piece can easily be excised for microscopic examination, this procedure should be adopted.

More than one tongue has been excised with the idea that it was the seat of cancer for want of appreciation by the surgeon of these additional and essential diagnostic methods.

Actinomycosis.

Though this disease is not common in the human subject it should be borne in mind (1) that its most frequent avenue of entrance is the mouth, (2) that the only positive diagnostic evidence is the presence of the actinomyces, (3) that the occurrence of this disease is diagnosed absolutely by its yellow granules visible to the naked eye, greasy to the touch, which, when placed under the microscope, reveal the distinctive characters of the growth.

I have seen these points revealed after the tongue had been removed for a lesion which was not too extensive for the remedial processes of scraping, caustice and iodic treatment. A biopsy for microscopic diágnosis priov to operation would hase eliminated such a serious error.

Treatment is by mouth washes, progressive increasing doses of iodides, iodoglidine, etc., and locally by iodex, etc. 